\title{
POLÍTICA PÚBLICA MOÇAMBICANA SOBRE A INCLUSÃO
}

Celestino Joanguete

Universidade do Minho, Braga

celestino.joanguete@gmail.com

Material original autorizado para su primera publicación en la revista

académica REDMARKA. Revista Digital de Marketing Aplicado

https://doi.org/10.17979/redma.2011.03.07.4739

Recibido: 14 Noviembre 2011

Aceptado 30 Noviembre 2011

\section{Abstract \\ MOZAMBICAN PUBLIC POLICY FOR DIGITAL INCLUSION}

According to the Population Census of 2007, from a total population of 20 million Mozambicans, only a reduced number of citizens living in urban areas benefits from knowledge of IT tools and the Internet. But, with a view to exposing more Mozambicans to the IT society the Government must create a set of projects and programs aimed at introducing more citizens to the new society based on IT. These public policies are considered as a way to promote active citizenship which in turn will lead to social inclusion through the introduction of the Internet in organizations, schools, government, etc.

The elaboration of the present text results from the bibliographical analysis of reports and studies published on the current period of training of the Technologies of Information and Communication in Mozambique as well as the rate of penetration and appropriation for the communities. The results of this analysis allowed researchers to arrive at the following conclusions: public policy on digital inclusion had a tremendous pull forward in the initial stages essentially after the 90s. There was, however, a slow-down in the process after that. 
Nowadays some projects on digital inclusion have declared bankruptcy mainly in education, health and the involvement of agricultural communities.

These remarks show that the exaggerated optimism on the part of the Government of Mozambique regarding IT makes it difficult to adopt a holistic approach to problems related to the development of real policies on digital inclusion, which would go beyond urban inclusion and encompass more rural areas which is where the majority of Mozambicans live.

Of these illations allow-in pointing out them that the exaggerated optimism of the government in relation to the technologies, makes it difficult a holistic share of Reals politics of digital inclusion, that not only see the inclusion side urban, but the agricultural regions that they constitute the thick number of the mozambican population, therefore is there that if it must start to construct the active and participativa citizenship in the society, fact that requires the knowledge of Reals necessities of this population segment

Key words: digital inclusion, ICT, strengthening of the citizen.

\section{Resumo}

Dos 20 milhões da população moçambicana, segundo o Censo Populacional de 2007, um número reduzido de cidadãos que moram nas zonas urbanas beneficiam de conhecimento das ferramentas informáticas e da Internet. Mas com vista a colocar a população moçambicana na sociedade de informação e de conhecimento, o governo deve criar um conjunto de projectos e programas com vista a incluir o cidadão na nova sociedade baseada na informação. Estas políticas públicas são vistas como meio de promoção de cidadania activa que leva à inclusão social através da Internet nas organizações, escolas, governo, etc.

A elaboração do presente texto resulta da análise bibliográfica de relatórios e estudos publicados sobre o actual estágio das Tecnologias de Informação e Comunicação em Moçambique bem como as suas taxas de penetração e apropriação pelas comunidades. Os resultados desta análise permitem-nos 
chegar a seguinte conclusão: as políticas públicas de inclusão digital tiveram um avanço extraordinário nos primeiros momentos, década 90, depois foram perdendo o seu alento ao longo dos anos. Hoje, apresentam-se alguns projectos de inclusão digital falidos, sobretudo no sector da educação, da saúde, e a nível das comunidades rurais.

Destas ilações permite-nos salientar que o optimismo exagerado do governo em relação às tecnologias, dificulta uma acção holística das reais políticas de inclusão digital, que vejam não só o lado urbano de inclusão, mas as regiões rurais que constituem o grosso número da população moçambicana, pois é lá que se deve começar a construir a cidadania activa e participativa na sociedade, facto que requer o conhecimento das reais necessidades deste segmento populacional

Palavra-chaves: inclusão digital, TIC, fortalecimento do cidadão. 


\section{Conceptualização}

A definição da inclusão digital não se limita apenas a literacia informática, disponibilizando computadores as comunidades e ensinando-as a manipular os sistemas operativos, embora isto também seja necessário. Portanto, a inclusão digital deve ser entendido como o acesso para todos às TIC incluindo a ligação à Internet, intervenção na esfera pública, realização de trabalhos e comunicação com a finalidade de realizar melhor as suas actividades diárias, (Sangonet: 2009: 36).

Para Costa $(S / D)$ a palavra "inclusão digital" pode ter vários significados. Por um lado pode significar inclusão dos que se sentem bloqueados, por outro, os analfabetos digitais.. Seja como for, neste contexto de estudo, entende-se inclusão digital como sendo uma forma de apoio ao cidadão na perspectiva de se integrar na sociedade de informação, sobretudo de cidadãos de poucas rendas.

De acordo com Costa (S/D) existem três categorias de inclusão digital: Técnica, cognitiva e económica. A inclusão técnica diz respeito à capacidade de manuseamento do computador, dos software e do acesso à Internet; a cognitiva é relacionada com autonomia e independência no uso complexo das TIC, visão crítica dos meios, prática social transformadora e capacidade de compreender os desafios da sociedade contemporânea e, a inclusão económica tem a ver com a capacidade financeira em adquirir e manter computador e custo para o acesso à Internet.

A inclusão deve ser entendida também como um impulso induzido e espontânea. Costa $(\mathrm{s} / \mathrm{d})$ dá o exemplo de criação de telecentros como o processo de inclusão induzida, enquanto a inclusão espontânea compreende as formas de acesso e uso de TIC

\section{A política nacional de informática}


Tal como noutros países, a entrada de Moçambique na Sociedade de Informação começou na década 90 com os primeiros serviços de Internet dialup fornecidos pelo Centro de Informática da Universidade Eduardo Mondlane , CIUEM, a partir de 1993.

O modelo de inclusão digital promovido pela política pública moçambicana tem sido a promoção de competências e manuseamento de ferramentas informáticas. Para tal, são mobilizados alguns actores nacionais como, por exemplo, a Comissão para a Política Informática, CPI, uma unidade criada pelo governo para desenhar a política informática de moçambique; Ministério da Ciência e Tecnologia, MCT, Ministério dos transportes e Comunicações, MCT; Unidade Técnica de implementação da Política de informática, UTICT, encarregue na informatização do sector público; Unidade Técnica de Reforma da Administração Financeira do Estado, UTRAFE; Instituto Nacional de Comunicações de Moçambique, INCM, Gabinete de Informação, GABINFO, instituição ligada ao Gabinete do Primeiro Ministro encarregue em registo dos meios de comunicação social; e Universidade Eduardo Mondlane, UEM, a primeira universidade pública (Sangonet, 2009).

As principais tarefas dos actores de política nacional de informática consistiam em: impulsionar e supervisionar a elaboração da Política de Informática; formulação e coordenação de Políticas e Estratégias de Ciência e Tecnologias; normalização e regulamentação ; promoção da valorização de conhecimento local ; protecção de direitos de propriedade; elaboração de Políticas de Comunicação e de Acesso Universal; implementação da Política Informática, elaboração de projectos de legislação e regulamentação; regulara a interligação das redes e a interoperacionalidade dos serviços públicos de telecomunicações, atribuição de licenças; garantir o apoio ao desenvolvimento do sector de comunicação social em Moçambique e formação de quadros superiores do sector das TIC, ( Sangonet, 2009: 6-9).

De acordo com o documento produzido pela Sangonet (2009), uma organização da sociedade civil sedeada na República da África do Sul, Moçambique foi um dos pioneiros a nível africano a reconhecer a importância 
dos uso das TIC para promover o desenvolvimento (Sangonet, 2009:9). Porém, o entusiasmo do Estado em relação a TIC foi esmorecendo ao longo dos anos a ponto de não constituir agenda prioritária do governo.

Apesar do reconhecimento da importância revolucionária da TIC na governação, no envolvimento do cidadão na participação política e na agilização dos processos de trabalhos e prestação de serviços público, as tecnologias são bem vistas em todos os discursos políticos como um dos factores básicos de desenvolvimento e de combate à pobreza e melhoramento das condições de vida dos moçambicanos.

Analisando os documentos do governo nas matérias de inclusão digital com particular enfoque para a "Política de Ciência e Tecnologia", divulgado em 2003 e para a "Estratégia de Ciência, Tecnologia e Inovação em Moçambique", publicado em 2006 com o horizonte temporal de 2006, nota-se nestes dois documentos o grande interesse do governo em criar infraestruturas que irão permitir o acesso das TIC em grande escala nacional.

Apesar da projecção de vontade, a maior parte dos projectos de inclusão digital com metas até 2005 ainda estão até hoje em fase de implementação, apenas houve pequenos avanços em 2006-2007. Esta lentidão deve-se, em parte, ao excesso de optimismo por parte dos principais actores de implementação de política nacional de inclusão digital, ou mesmo da falta de linha de orientação e de prioridades.

Além de tudo, nota-se que a política nacional de inclusão digital é bastante generalista e concentradora. O grande foco vai para o aspecto de governação electrónica no sector público, mas ainda falta uma política sectorial das TIC como educação, comércio, media e das comunidades rurais.

As infra-estruturas adequadas, a capacitação humana, parcerias e mobilização de recursos para entrada na Sociedade de Informação são fundamentais para as organizações e pessoas singulares. Mas ainda falta uma política de capacitação de recursos humanos e do fortalecimento do cidadão em matérias 
das tecnologias, salvo alguns casos isolados que são assegurados por alguns institutos e universidades, mas é necessário desenvolver políticas de capacitação extensiva para as comunidades do interior. Para tal requer-se a criação de infra-estruturas de conectividade nos distritos quer para a governação electrónica, educação, telecomunicações, operadores de rádio, televisão e do comércio. .

Segundo Sangonet (2009), para suprir o fosso digital interno, Moçambique deve assumir três desafios fundamentais para a efectiva inclusão digital da comunidade moçambicana: Infra-estruturas, capacitação humana, custos e preços.

i) Infra-estruras físicas são a base sobre a qual assenta a política de inclusão digital. Em Moçambique a conectividade e a comunicação de dados é extremamente lenta, oscilação e quedas constantes. Ainda mais, as infraestruturas de telecomunicações não estão distribuídas pelo país de forma equitativa, mas concentram-se mais nas grandes urbes, oferecendo menos oportunidade de acesso para a maioria da população. $O$ acesso às infraestruturas de telecomunicações depende da existência de energia, mas a fraca qualidade da energia fornecida também constitui factor de impedimento. Para isso, é necessário estender a comunicação via banda larga, promover s regulamentação de partilha de infra-estruturas; assegurar a igualdade de acesso, reduzir os preços dos computadores pessoais e apoiar os projectos de difusão e acesso às TIC para pessoas portadoras de deficiência.

ii) Capacitação humana. Tendo em conta que o grosso número da população moçambicana situa-se na zona rural, que neste momento assume com naturalidade que os computadores e a Internet são para pessoas ricas ou com qualificações académicas mais alta, isto faz com que com que os recursos e as oportunidades cheguem primeiro as grandes cidades onde estão concentradas as camadas intelectuais e novos ricos e só depois à comunidade rural. Face a esta realidade, o desafio principal reside na massificação de formação, que vai para além de cursos básicos, mas para a capacitação do uso efectivo de TIC por cada grupo-alvo: homens e mulheres; 
aposta no sistema nacional de educação como agente promotor de inclusão digital; capacitação dos professores, não só nas habilidades básicas mas sobretudo no uso de TIC no processo de ensino-aprendizagem.

iii) O preço da Internet de banda larga em Moçambique é exorbitante, sobretudo para a população rural que vive com menos de 1 Dólar por dia. Por isso, o primeiro desafio é baixar o custo da Internet junto dos operadores ou assegurar que os usuários beneficiem de preços reduzidos.

De acordo com o documento da Organização para Cooperação e Desenvolvimento Económico, OCDE, de 2008, sobre a educação, relata no seu texto que a banda larga é o factor impulsionador da mudança estrutural, criação de novos serviços digitais, aumenta a eficiência das empresas, melhora a concorrência e sustenta a globalização:

A banda larga e as aplicações a ela associadas contribuem para a transformação da actividade económica como o fizeram outras tecnologias de utilização geral, como a electricidade e o motor de combustão interna. Os impactos da banda larga podem ser mais importantes à medida que o preço das TIC baixar mais dramaticamente. Ainda que necessários, os investimentos complementares em inovações de competências e organizacionais podem levar algum tempo a materializarem-se para permitir a contribuição da banda larga no crescimento e na criação de emprego (OCDE, 2008: 8)

A largura da banda constitui o ponto fraco do governo por várias razões: a falta de infra-estruturas de conectividade e as taxas exorbitantes cobradas pelos operadores internacionais de fornecimento de serviços da Banda Larga.

\section{Fortalecimento do cidadão}


A capacitação de recursos humanos em TIC abre espaço para formação de uma nova cidadania mais pró-activa. Assim sendo, é condição essencial para o desenvolvimento de recursos humanos em três níveis: primeiro, assegurar que todos os cidadãos tenham conhecimentos básicos para o uso das TIC para diversos fins, segundo, assegurar a formação de novas gerações de cidadãos capazes de liderar e sustentar a evolução tecnológica e, por último, formação de novos usuários conectados às redes mundiais de troca de informação e de conhecimento.

Muitas iniciativas têm sido tomadas para a criação de condições básicas de inclusão digital:

- $\quad$ Projecto SchoolNet- uma iniciativa da UEM que a partir de 1997 passou para o Ministério da Educação e Cultura, MEC, em 2002. O projecto tinha em vista a introdução de salas de informática nas escolas, começando a nível pré-universitário e nos centros de formação dos professores, passando progressivamente para o ensino secundário geral e primário. Actualmente, 22 institutos de formação de professores e 76 escolas secundárias e médias estão equipadas, mas devido aos altos custos o número de centros de formação de professores e das escolas secundarias nas capitais provinciais e grandes cidades com acesso a Internet é reduzido.

- Introdução da disciplina de informática no tronco comum do currículo escolar, cujo arranque deve se concretizar em 2009-2010

- $\quad$ Adopção de curriculum certificado de TIC para o desenvolvimento. Este projecto foi adoptado pela UTICT a partir da European Computer Driving Licence (ECDL)

- Formação dos formadores e a sua colocação em instituições vitais , com destaque para instituições de formação de professores e funcionários de centros de formação de professores.

- Cursos básicos de informática, 
- Estabelecimento de rede Moçambicana de ensino Superior e de pesquisa ( MoReNet) pelo Ministério da Ciência e Tecnologia que ligará 25 instituições superiores numa rede da Internet de alta velocidade, visando transformar qualitativamente e quantitativamente a capacidade de busca e partilha de conhecimento, cooperação nacional e internacional em projectos de pesquisa e acesso a recursos como a biblioteca e ferramentas de ensinoaprendizagem.

- $\quad$ Acesso a educação por via de sistema de ensino à distância (EAD) usando TIC a todos os níveis, começando pelo ensino superior.

No que se refere às infra-estruturas básicas estão sendo criadas as seguintes acções:

- Rede Nacional de transmissão das Telecomunicações de Moçambique de banda larga e com cobertura nacional usando o cabo subterrâneo de fibraóptica

- Aumentar do número de cabos submarinos para acelerar o fluxo de informação digital dentro e fora do país,

- $\quad$ Expansão da rede eléctrica nacional para as localidades e distritos

- Instalação defrontes de energia alternativa nos distritos

- $\quad$ Expansão da rede de telefonia móvel

Estes são considerados alguns avanços feitos pelo governo para a implementação da estratégia sobre TIC., mas a capacitação humana, de acordo com SangoNet (2010), é condição essencial para o desenvolvimento.

As tabelas que se seguem ilustram claramente o estágio da penetração da TIC em vários substratos da sociedade moçambicana.

Tabela no 1: Percentagem de cobertura dos serviços TIC por província 


\begin{tabular}{|c|c|c|c|c|}
\hline Província & E-mail & Internet & $\begin{array}{l}\text { telefonia } \\
\text { fixa }\end{array}$ & Telefonia móvel \\
\hline Maputo & & 37.5 & 87.5 & 100 \\
\hline Maputo & & & & \\
\hline Cidade & & 100 & 100 & 100 \\
\hline Gaza & & 16.7 & 50 & 58.3 \\
\hline Inhambane & & 21.4 & 85.7 & 78.6 \\
\hline Manica & & 20 & 60 & 60 \\
\hline Sofala & & 8 & 84.6 & 61.5 \\
\hline Tete & & 8 & 61.5 & 38.5 \\
\hline Zambézia & & 18 & 100 & 58.8 \\
\hline Nampula & & 23.8 & 90.5 & 61.9 \\
\hline Niassa & & 13 & 43.8 & 18.8 \\
\hline Cabo Delgado & & 18 & 100 & 41.2 \\
\hline
\end{tabular}

Fonte. MCT, Indicador de Ciência e Tecnologia, 2009

Relativamente a percentagem de cobertura dos serviços de TICs por província, há a destacar que no serviço de Internet Maputo Cidade possui uma cobertura de $100.0 \%$, seguido de Maputo Província com 37.5\%, Nampula (23.8\%), Inhambane (21.4\%) e Manica (20\%), as províncias com baixa cobertura são de Sofala e Tete com $8.0 \%$ respectivamente. As restantes províncias situam-se na banda dos $13-18 \%$.

Estes dados revelam que as grandes capitais provinciais são as mais privilegiadas em termos de acesso às tecnologias básicas de comunicação e 
informação, ou seja, telefone e Internet, porém não existem informações representativas do estado da situação das comunidades rurais moçambicanas

Tabela no 2: Acessibilidade Familiar às TIC

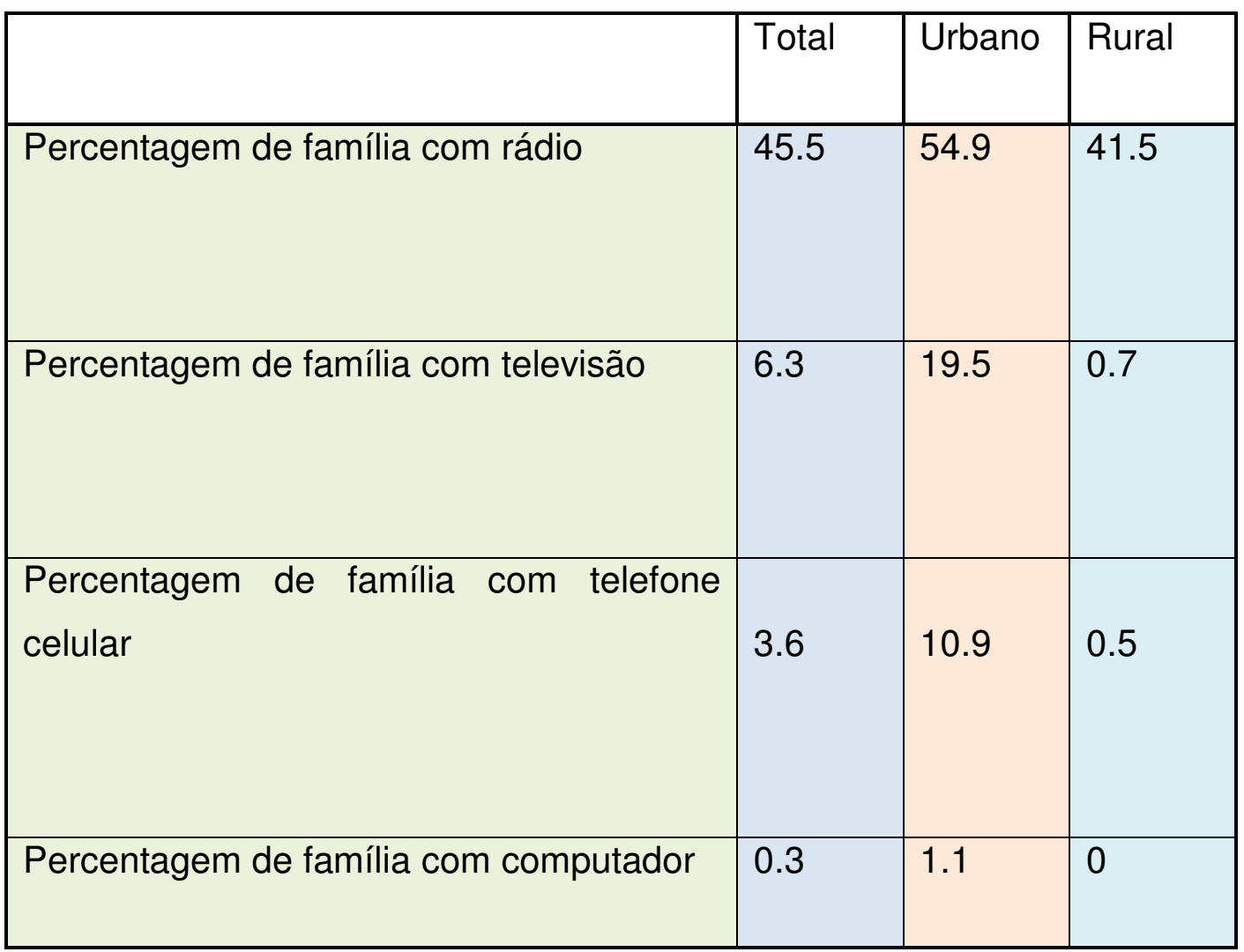

Fonte. MCT, Indicador de Ciência e Tecnologia, 2009

Os benefícios da TIC na vida do cidadão vão muito além de habilidade de manipulação das ferramentas, mas inclui benefícios económicos, melhoramento da qualidade de vida e bem-estar dos cidadãos, aquisição de novos conhecimentos e integração na rede mundial de troca de informação e de experiências diversas e de forma transversal, os benefícios se estendem para a expansão da liberdade dos indivíduo, sobretudo de expressão e de livre comunicação.

A tabela revela que $41.5 \%$ da população rural apenas tem a rádio como meio de acesso a informação. Quanto à televisão, celular e computador, a população rural não tem acesso a estes recursos tecnológicos. 
Tabela no3: Escolas com facilidades a TIC em Moçambique

\begin{tabular}{|c|c|c|}
\hline Tipo de escola & $\begin{array}{l}\text { № total de escolas } \\
\text { públicas }\end{array}$ & $\begin{array}{l}\% \text { escolas com salas de } \\
\text { informática }\end{array}$ \\
\hline $\begin{array}{l}\text { Ensino geral primário } 1^{\circ} \\
\text { nível }\end{array}$ & 8700 & 0,02 \\
\hline $\begin{array}{l}\text { Ensino Geral Primário } \\
2^{\circ} \text { nível }\end{array}$ & 1320 & 1.1 \\
\hline $\begin{array}{l}\text { Ensino Geral } \\
\text { Secundário } 1^{\circ} \text { Ciclo }\end{array}$ & 156 & 9.6 \\
\hline $\begin{array}{l}\text { Ensino Geral } \\
\text { Secundário } 2^{\circ} \text { Ciclo }\end{array}$ & 35 & 91.4 \\
\hline
\end{tabular}

Fonte: MCT, Indicador de Ciência e Tecnologia, 2009

O nível de utilização da TIC nas escolas públicas moçambicanas ainda está muito longe de conduzir o cidadão a uma inclusão digital. A fase de iniciação escolar, ou seja, o ensino primário do $1^{\circ}$ e $2^{\circ}$ grau apresenta uma taxa reduzida de salas de informática para o ensino do computador. Sendo estas duas primeiras fases fundamentais para familiarizar as crianças com 0 computador, apresentam uma taxa de $1.2 \%$. 
Tabela no 4: Computadores nas escolas públicas

\begin{tabular}{|l|c|}
\hline Províncias & № computadores \\
\hline Maputo & 137 \\
\hline Maputo cidade & 342 \\
\hline Gaza & 74 \\
\hline Inhambane & 111 \\
\hline Manica & 61 \\
\hline Sofala & 115 \\
\hline Tete & 100 \\
\hline Zambézia & 96 \\
\hline
\end{tabular}

REDMARKA UIMA-Universidad de A Coruña - CIECID

Año IV, Número 7, (2011), v3 pp. 61-82

http://www.redmarka.org/

ISSN 1852-2300 


\begin{tabular}{|l|c|} 
Nampula & 113 \\
\hline Niassa & 63 \\
\hline Cabo delgado & 103 \\
\hline Total & 1315 \\
\hline
\end{tabular}

Fonte: MCT, Indicador de Ciência e Tecnologia, 2009

Computadores e a Internet são ainda menos disseminados em todo o país. Havia uma estimativa de 100.000 usuários de computadores em todo país em 2007, na sua maioria homens de renda mais alta, nas áreas urbanas. Havia estimativa de 24000 usuários de Internet em 2009, e este número estava circunscrito nos grupos de maior estatuto social. Mas este número está a aumentar. Contudo, a falta de acesso a computadores e à Internet coloca desafios para o desenvolvimento do país( Panos, 2010)

A partir da leitura de outros documentos e complementando com as tabelas acima indicadas, conclui-se que, dentre vários avanços, destaca-se a elaboração e aprovação da Estratégia de Governo Electrónico, Desenvolvimento do Portal do Governo de Moçambique que conta com mais de 2 milhões de visitantes por mês, interligação de 128 instituições através da Rede Electrónica do Governo (GovNet-órgãos centrais e provinciais), desenvolvimento do Sistema Centralizado de Correio Electrónico do Governo com mais de 3.000 utilizadores, criação da Plataforma Dzowo como base de dados e de interacção electrónica dos pesquisadores moçambicanos e seus parceiros internacionais, estabelecimento do Sistema de Administração Financeira do Estado (e-SISTAFE) pelo Ministério das Finanças.

O Sistema de Cadastro e Sistema de Registo e Licenciamento de Empresas (SISCAL) pelo Ministério da Indústria e Comércio, o sistema de informação de gestão da terra pelo Ministério da Agricultura, o sistema de informação de gestão da nova carta de condução pelo Ministério de Transportes e Comunicação, o sistema de informação de gestão da emissão do registo 
criminal pelo Ministério da Justiça, o Sistema Electrónico de Cadastro de Agentes e Funcionários do Estado (eCAF) pelo Ministério da Função Pública, desenvolvimento do projecto do Número Único de Identificação do Cidadão (NUIC), desenvolvimento do Projecto MorNet que visa interligar, em rede de internet, Instituições de Investigações e de ensino superior, elaboração e aprovação da Política de Telecomunicações e a Lei de Transacções Electrónicas, modernização da infra-estrutura de telecomunicações com a utilização da fibra óptica nas comunicações, elaboração do Museu Virtual de Açúcar, estabelecimento de 17 Centros Multimédia Comunitários (CMCs), entre várias acções de implementação de programas de implementação de inclusão digital, fazem parte do projecto governamental do fortalecimento das infraestruturas da TIC.

A disseminação da TIC em Moçambique é orientada para as infra-estruturas, que de certo modo apresentam as suas precariedades e aliada à pobreza da maioria da população rural que não pode ter acesso dos mesmos e, sobretudo, o grosso número das populações rurais não possui rede de energia eléctrica nem comunicações por telefone fixo.

O grande sucesso das TIC tem sido os telefones celulares. Até 2008, segundo o estudo da SangoNet em 2009, havia mais de quatro milhões de assinantes de telefonia móvel no país, o equivalente a 21 porcento da população moçambicana e o seu número foi crescendo cerca de 50 porcento ao ano. Pessoas de todos os grupos sociais, incluindo os pobres, viram o valor dos telefones celulares nas comunicações quotidianas.

Nota-se que a rádio continua a desempenhar o papel preponderante nas populações urbanas e rurais. 45.5 porcento de agregados familiares das áreas rurais e urbanas dispõe de aparelho receptor. Dai que a rádio é o principal canal de informação, graças à desregulamentação e da legislação sobre a liberdade de imprensa. 
O reduzido número de televisões nas populações rurais deve-se a dois factores: são caras e precisam de energia que não é disponível nas áreas rurais.

Apesar dos esforços de inclusão digital ainda prevalecem muitos constrangimentos a nível do governo para levar a cabo o compromisso nacional de expansão da política de TIC. Os constramgimentos tem a ver com a falta uma coordenação interna nos sectores do Estado para implementação de políticas de TIC; os graduados das universidades não reúnem habilidades e nem domínio suficiente do computador nas ferramentas básicas; o avanço das comunicações móveis e a consequente convergência dos meios que facilitarão a produção e partilha de informação não fossem matérias de regulamentação como forma de defesa de livre circulação de informação na Internet.

A TIC tem implicações directas para os media, particularmente, quando obriga a migração do analógico para o digital. O sistema digital oferece múltiplas vantagens para produtores e usuários de conteúdos em termos de qualidade melhorada de som e imagem, para rádio e televisão; distribuição de conteúdos para diversas plataformas de recepção de conteúdos, para os meios impressos.

Neste cenário de migração tecnológica, o governo ainda não tomou decisões importantes no sentido de garantir a defesa de interesse público, no que concerne à regulação dos novos sistemas de rádio e televisão, licenciamento de operadores, padrão de qualidade, custos, etc

\section{O desafio da inclusão das línguas moçambicanas}

Moçambique é um país constituído um pouco mais de 23 linguas nacionais e de uma grande diversidade cultural. Constitucionalmente a língua portuguesa é consagrada como a língua oficial, mas coexiste com a língua oficial outras muitas outras africanas de origem Bantu, que constituem o grosso número do quadro linguístico nacional. 
A pluridade linguística é a característica cultural do país, desde o Norte até ao Sul. De acordo com Ngunga (2011), para a maioria da população, as línguas moçambicanas constituem as suas línguas maternas e instrumento de comunicação diária.

Segundo os resultados do Censo populacional de 2007, Emakhua é a língua moçambicana mais falada em Moçambique com $26.3 \%$ falantes, seguida de Xichangana com $11.4 \%$, Elomwe com 7.9\% ; Cisena com 7.0 e as outras línguas assumem $41.0 \%$, excepto a lingua portuguesa, que é falada por $6.5 \%$ da população moçambicana que vive nas grandes urbes e que adoptam-na como a língua falada em casa. Assim ilustra-nos a tabela que se segue:

Tabela 5: Distribuição percentual da população de 5 anos e mais por grandes grupos de idade segundo a língua materna

\begin{tabular}{|c|c|c|c|c|}
\hline \multirow{2}{*}{ Língua materna } & \multirow{2}{*}{ Total } & \multicolumn{3}{|c|}{ Grupos de idade } \\
\hline & & $5-19$ & $20-49$ & $50+$ \\
\hline$N(000)$ & $12,536.8$ & $5,680.1$ & $5,430.6$ & $1,426.1$ \\
\hline Total & 100.0 & 100.0 & 100.0 & 100.0 \\
\hline Português & 6.5 & 8.5 & 5.5 & 2.1 \\
\hline Emakhuwa & 26.3 & 24.8 & 28.0 & 25.6 \\
\hline Xichangana & 11.4 & 11.8 & 10.2 & 14.1 \\
\hline Elomwe & 7.9 & 7.4 & 8.6 & 6.8 \\
\hline Cisena & 7.0 & 6.9 & 7.2 & 6.7 \\
\hline
\end{tabular}




\begin{tabular}{|r|r|r|r|r|}
\hline Echuwabo & 6.3 & 6.1 & 6.6 & 5.9 \\
\hline $\begin{array}{r}\text { Outras línguas } \\
\text { moçambicanas }\end{array}$ & 33.0 & 32.7 & 32.2 & 37.5 \\
\hline Outras línguas estrangeiras & 0.4 & 0.3 & 0.5 & 0.6 \\
\hline Nenhuma & 0.1 & 0.1 & 0.1 & 0.0 \\
\hline Desconhecida & 1.3 & 1.5 & 1.2 & 0.7 \\
\hline
\end{tabular}

Analizando a tabela acima representada, os dados revelam que cerca de $90 \%$ de moçambicanos são falantes das línguas nacionais, o que significa que elas não são línguas alternativas na comunicação entre os moçambicanos, mas constituem, por si só , os principais meios de comunicação através dos quais os moçambicanos se comunicam.

De acordo com Ngunga(2011), as práticas linguísticas oficiais em Moçambique apresentam grandes paradoxos em relação à situação linguística do país acima descrita. São claras as disparidades numéricas dos falantes da língua portuguesa por todo o território nacional. A tabela que se segue, apresenta 0 referido paradoxo em termos de distribuição de falantes da língua portuguesa em cada província moçambicana e na Cidade de Maputo (capital do País):

Table 6: Falantes de Português das dez províncias e da Cidade de Maputo.

\begin{tabular}{|l|r|r|r|}
\hline Província & População total & $\begin{array}{r}\text { Falantes de } \\
\text { Português }\end{array}$ & $\%$ \\
\hline
\end{tabular}




\begin{tabular}{|r|l|r|r|r|}
\hline 1 & Cidade de Maputo & 959.474 & 412.162 & 43.0 \\
\hline 2 & Maputo Province & 1.025 .871 & 283.665 & 27.7 \\
\hline 3 & Sofala & 1.338 .709 & 177.655 & 13.3 \\
\hline 4 & Zambézia & 3.021 .246 & 277.906 & 9.2 \\
\hline 6 & Nampula & 3.183 .399 & 277.223 & 8.7 \\
\hline 7 & Manica & 904.784 & 61.223 & 6.8 \\
\hline 8 & Inhambane & 1.131 .269 & & 5.7 \\
\hline 9 & Gaza & 1.058 .135 & 54.057 & 5.4 \\
\hline 10 & Cabo Delgado & 1.024 .911 & & \\
\hline & & & & \\
\hline
\end{tabular}

Fonte: Ngunga (2011).

A distribuição percentual de falantes da língua portuguesa pelas dez províncias moçambicanas e incluindo a capital do País relativamente à totalidade dos habitantes das respectivas unidades territoriais, indica que os falantes da língua oficial portuguesa situam-se na ordem de 50 porcento, grosso número deles concentrados nas três províncias , Maputo-cidade, Maputo província e Sofala.

Portanto, estas percentagens permitem deduzir que $90 \%$ da população moçambicana não tem o domínio da língua portuguesa. Apenas 10\% de habitantes de Moçambique, distribuídos de forma não muito expressiva, falam a língua portuguesa. 
A política de inclusão digital, os acelerados avanços tecnológicos, a migração dos conteúdos dos jornais para as plataforma digital, irão acentuar ainda mais esta contrariedade, o que significa que $89.5 \%$ da população moçambicana que fala as línguas moçambicanas, sem ter tido nenhum contacto com a língua portuguesa, será os próximos info-excluido do processo de inclusão digital.

Na perspectiva do consumo dos produtos noticiosos, Ngunga (2011) afirma que Moçambique conta actualmente com cerca de 500 órgãos de comunicação social dos quais cerca de 80 (16\%) usam línguas moçambicanas. Destes 80 órgãos, 98.8\% são rádios comunitárias. Para Ngunga, se a comunicação social desempenha o papel importante para o desenvolvimento, é legítimo concluir que a língua portuguesa "funciona como meio de exclusão dos moçambicanos nesse processo. Portanto, ela só serve para $10 \%$ de moçambicanos no acesso à informação.

\section{Conclusão}

A inclusão digital não deve ser vista exclusivamente na perspectiva infraestrutural, pois a componente de desenvolvimento social deve fazer arte integrante.

O acesso aos media digitais não é uma exclusividade da elite. Há várias formas de melhorar o cenário actual de exclusão, em relação ao campo/cidades. A instalação de centros de fortalecimento do cidadão , computadores no ensino, uso dos media como metodologia do ensino e aprendizagem, são alternativas que se mostram mundialmente eficientes nos países em desenvolvimento, desde que sejam incluídas nas políticas públicas de inclusão digital, com formadores competentes, equipamentos tecnológicos adequados, conectividade às redes mundiais de comunicação, legislação favorável, etc..

O ensino público devia assumir a dianteira no uso das tecnologias, principalmente, nos primeiros anos do ensino básico. Caso contrário, o sistema nacional do ensino público estará a criar um fosso digital entre o 
público e o privado. Num mundo em constantes mudanças, onde a Internet é o meio de ligação mundial às redes de conhecimento e de informação, não se pode privar as novas gerações para o isolamento, tanto do ponto de vista individual como da perspectiva da sociedade.

\section{Bibliografia}

Costa, Leonardo.(S/D),"Inclusão digital : uma alternativa para o social? Análise de projectos realizados em Salvador", In Revista Brasileira de Inovação Científica em Comunicação, Vol. 1, nº 1, pp. 107-122, [consultado no dia 27/09/11],no

http://www.portcom.intercom.org.br/ojs-2.3.12/index.php/inovcom/article/view/310

OCDE, (2008) Tecnologias de Informação e Comunicação Perspectivas da Tecnologia de Informação da OCDE 2008

MCT, (2009), Indicadores de Ciência e Tecnologia de Moçambique, Maputo

Sangonet, (2009) Inclusão Digital em Moçambique: um desafio para todos, relatório de Moçambique, CIUEM.

Martini, Renato P.P. (2005), INCLUSÃO DIGITAL \& INCLUSÃO SOCIAL, Inclusão Social V. 1. № 1, [consultado no dia 25/10/11] no http://revista.ibict.br/inclusao/index.php/inclusao/article/view/7/13,

MCT, (2009), Indicadores de Ciência e Tecnologia de Moçambique, Maputo MCT , (2003): Política de Ciência e Tecnologia, Ministério de Ciência e Tecnologia , Maputo, ,[on line]

http://www.mct.gov.mz/pls/portal/docs/PAGE/PORTALCIENCIATECNOLOGIA/ PUBLICACOES/POLITC T 0.PDF, consultado no dia 08/10/2010

Para citar este artículo:

Joanguete, Celestino (15-12-2011). POLÍTICA PÚBLICA MOÇAMBICANA SOBRE A INCLUSÃO DIGITAL.

REDMARKA - CIECID - Unidad de Investigación en Marketing Aplicado-Universidad de A Coruña

Año IV, Número 7, V3, pp.61-82

ISSN 1852-2300

URL del Documento : cienciared.com.ar/ra/doc.php? $n=1603$

URL de la Revista : cienciared.com.ar/ra/revista. php?wid=39 